\title{
Anaerobic Bacteremia: Incidence, Patient Characteristics, and Clinical Significance
}

\author{
Donald P. Lombardi, M.D., N. CARYENGLEBERG, M.D., Ann Arbor, Michigan
}

PURPOSE: In the 1970s, blood culture for obligate anaerobic bacteria became routine in most United States hospitals. Since then, various authorities have reported isolation of obligate anaerobes in $5 \%$ to $25 \%$ of blood cultures. Our experience suggests a much lower frequency; therefore, we retrospectively assessed the occurrence and significance of these cultures at our institutions.

PATIENTS AND METHODS: Sixty-six patients at the University of Michigan Hospitals (UMH) and nine patients at the Ann Arbor Veteran's Administration Medical Center (AAVAMC) had one or more blood cultures positive for an obligate anaerobe between July 1, 1987, and December 31, 1988. Their medical records were reviewed retrospectively.

RESULTS: The proportion of positive blood cultures yielding obligate anaerobes was $3.2 \%$ at the UMH and $1.8 \%$ at the AAVAMC. The incidences of clinically significant anaerobic bacteremia at the two hospitals were 0.68 and 0.54 cases per 1,000 patient admissions. Among the 40 patients from whom significant isolates were obtained, 15 (38\%) had a fatal outcome. Bacteroides and Clostridium species accounted for $90 \%$ of the isolates and all of the fatal cases. 'The source for anaerobic bacteremia was usually obvious; 30 of the 40 patients were given empiric antibiotic therapy for anaerobes. The gastrointestinal tract was the source in two thirds of the cases and was clearly implicated as the source of $80 \%$ of the fatal bacteremias.

CONCLUSIONS: The frequency of anaerobic bacteremia in our hospitals is much lower than was suggested in several large studies during the 1970 s, probably reflecting a real decline in the incidence. The clinical features of our cases are

From the Departments of Internal Medicine (DPL, NCE) and Microbiolagy and Immunology (NCE), University of Michigan Medical School, Ann Arbor, Michigan.

Requests for reprints should be addressed to N. Cary Engleberg, M.D. Department of Microbiology/Immunology, 6643 Medical Science-II, University of Michigan Medical School, Ann Arbor, Michigan 48109-0620.

Dr. Lombardi's current address is National Cancer Institute/Navy Medical Oncology Branch, Naval Hospital, Building 8, Room 5101, Bethesda 20889-5105

Manuscript submitted March 21, 1991, and accepted in revised form August 30, 1991 similar to those of previous studies, and the mortality is still high despite the use of antibiotics effective against anaerobes. Since most patients were thought to have anaerobic infections at the time that cultures were obtained, they were usually treated empirically. Subsequent blood cultures positive for anaerobes infrequently influenced clinical management.

D uring the late 1960s and early 1970 s, when improvements in isolation techniques for obligate anaerobes were introduced, several large studies drew attention to the potential significance of anaerobic bacteremias [1-6]. Subsequently, cultivation for strict anaerobes has become a routine part of the clinical evaluation for bacteremia in most large hospitals. The frequency of anaerobic bacteremia was estimated at-and is currently reported in four major textbooks of medicine to be -between 5\% and 25\% [7-10]. However, recent experience at our hospitals suggested a much lower isolation frequency than the widely quoted reports. Therefore, we undertook a retrospective chart review at the University of Michigan Hospitals (UMH) and the Ann Arbor Veteran's Administration Medical Center (AAVAMC) in order to evaluate the incidence and clinical features of anaerobic bacteremia and to reassess the impact of this diagnosis on patient management.

\section{PATIENTS AND METHODS}

UMH consists of six inpatient facilities (University Hospital, Mott Children's Hospital, Women's Hospital, Holden Prenatal Hospital, Kellogg Eye Center, and the Child Psychiatric Center) that provide tertiary health care for up to 888 patients. All six facilities are serviced by a single microbiology laboratory. At these facilities, blood samples are routinely cultured using both a lysis-centrifugation tube (DuPont Co., Wilmington, Delaware) for aerobes and an unvented vacuum bottle containing supplemented peptone broth (Becton Dickinson Vacutainer Systems, Rutherford, NJ) for aerobes and anaerobes. The peptone broth bottles with signs of growth are subcultured under aerobic and anaerobic conditions, and isolates are identified by standard methods. Bottles without signs of growth 
are subcultured aerobically and anaerobically on Days 3 and 6 and held for a total of 8 days incubation before they are discarded.

AAVAMC, a neighboring 488-bed referral center, utilizes the Bac'Tec (Becton Dickinson Diagnostic Instrument Systems, Towson, Maryland) automated radiometric sampling system for blood cultures. A blood culture "set" consists of two or three bottles, one for aerobes, one for anaerobes, and one for patients receiving antibiotics (BacTec $6 \mathrm{~B}, 7 \mathrm{D}$, and $16 \mathrm{~B}$ media, respectively). Any bottle having a significant growth index is subcultured anaerobically. Those having no detectable growth after 8 days are discarded. Blind subcultures are not routinely performed.

All positive blood cultures between July 1, 1987, and December 31,1988, recorded by the microbiology laboratories at the two hospitals were reviewed for study. Patients with obligate anaerobes isolated from their blood cultures were identified, and their medical records were reviewed. Charts were reviewed with attention to age, sex, clinical service, number of positive cultures, clinical presentation, probable portal of entry, antibiotic treatment, complications (e.g., jaundice, metastatic abscesses, thrombophlebitis), maximum temperature, white blood cell count, presence of septic shock, mortality, and temporal association with a surgical or invasive diagnostic procedure. Since we thought that the physicians caring for the patients were in the best position to assess whether a bacterial isolate from the blood was clinically significant, culture isolates were defined as "clinically significant" or "contaminants" based on the physician of record's judgment, either as stated in writing or as indicated by specific actions taken in response to the culture (i.e., specific therapy versus no therapy). In addition, each of the 75 cases was reviewed by us, and there was complete agreement in the assessment of the clinical significance of the cultures with the physician of record.

\section{RESULTS}

\section{Frequency and Incidence of Anaerobic Bacteremia}

During the 18-month period encompassed by our review, 48,645 patients were admitted to the UMH, and 31,758 blood cultures were drawn. Bacterial or fungal isolates were obtained from 2,712 blood culture sets $(8.5 \%)$. Eighty-six cultures $(3.2 \%$ of the positive cultures) from 66 patients yielded obligate anaerohes. However, only 51 of these anaerobic isolates (i.e., $59 \%$ of the blood cultures positive for obligate anaerobes) were clinically significant; the remainder were determined to be contaminants. The 51 significant isolates were cultured from 33 patients. 'Those patients with more than one posi- tive blood culture had the same isolate from multiple cultures. At the time of the bacteremia, most of these patients were on the Surgical and Medical Services (13 and 10 patients, respectively). The remaining cases occurred in patients on the Obstetrics/Gynecologic (4), Pediatric (3), Urologic (2), and Emergency (1) Services. The overall incidence of clinically significant anaerobic bacteremia at UMH was 0.68 cases per 1,000 hospital admissions.

During the same time period at the AAVAMC, 12,879 patients were admitted. A total of 6,594 blood cultures were obtained, of which 782 (11.9\%) were positive for bacteria or fungi. Fourteen of these cultures yielded obligate anaerobes $(1.8 \%$ of the positive blood cultures); however, only 12 of these (i.e., $86 \%$ of the anaerobe-positive blood cultures) were clinically significant isolates. These 12 significant cultures were obtained from seven patients; four were Medical Service inpatients, two were Surgical Service inpatients, and one patient was in the emergency room at the time the bacteremia developed. The overall incidence of clinically significant anaerobic bacteremia at the AAVAMC was 0.54 cases per 1,000 hospital admissions.

\section{Microbiology of Anaerobic Bacteremia}

A variety of obligate anaerobes were grown from the clinically significant blood cultures, although Bacteroides sp. accounted for $65 \%$ of the isolates (Table I). The two most common species were Bacteroides fragilis (from 12 patients) and Bacteroides thetaiotaomicron/ovatus group (from eight patients). Cultures from 11 patients yielded Clostridium sp., with Clostridium septicum and Clostridium perfringens isolated from four patients each. Three patients had Fusobacterium isolated from culture. One patient had polymicrobial anaerobic sepsis with $B$. fragilis and Fusobacterium mortiferum, and four patients had a facultative isolate, in addition to an anaerobic isolate, from blood cultures taken during the same septic episode. Most of the isolates from contaminated blood cultures were Proprionibacterium acnes. This species accounted for 30 of the 37 contaminant isolates that were taken from 29 patients (81\% of all anaerobic contaminants). Single isolates of Veillonella sp., peptostreptococcus, and $B$, distasonis, as well as two isolates each of $B$. melaninogenicus and Clostridium sp., were also judged to be contaminants by the physicians in charge.

\section{Clinical Presentation}

Patients with anaerobic bacteremia ranged from 7 to 82 years of age $($ mean $=54$ years; median $=59$ years). Males (17 patients) and females (16 patients) were equally affected at $\mathrm{UMH}$; all of the 


\section{TABLE I}

Clinically Significant Anaerobic Blood Culture Isolates at Two Ann Arbor Hospitals, July 1987 to December 1988

\begin{tabular}{|c|c|c|c|c|}
\hline \multirow[b]{2}{*}{ Species } & \multicolumn{2}{|c|}{ UMH } & \multicolumn{2}{|c|}{ AAVAMC } \\
\hline & $\begin{array}{l}\text { No. of } \\
\text { Isolates }\end{array}$ & $\begin{array}{l}\text { Patients and } \\
\text { Mortality* }\end{array}$ & $\begin{array}{l}\text { No. of } \\
\text { Isolates }\end{array}$ & $\begin{array}{l}\text { Patients and } \\
\text { Mortality }\end{array}$ \\
\hline $\begin{array}{l}\text { Bacteroides species } \\
\text { B. fragilis } \\
\text { B. thetaiotaomicron/ovatus } \\
\text { B. buccae } \\
\text { B. bivius } \\
\text { B. uniformis } \\
\text { B. asaccharolyticus }\end{array}$ & $\begin{array}{r}29 \\
13 \\
10 \\
4 \\
1 \\
1\end{array}$ & $\begin{array}{l}7 / 17^{\dagger} \\
3 / 8 \\
4 / 6 \\
0 / 1 \\
0 / 1 \\
0 / 1\end{array}$ & $\begin{array}{r}12 \\
8 \\
2\end{array}$ & $\begin{array}{l}3 / 7 \\
1 / 4 \\
2 / 2 \\
\\
0 / 1\end{array}$ \\
\hline $\begin{array}{l}\text { Clostridium species } \\
\text { C. perfringens } \\
\text { C. septicum } \\
\text { C. tertium } \\
\text { C. ramosum } \\
\text { C. clostridiforme }\end{array}$ & $\begin{array}{r}16 \\
6 \\
6 \\
2 \\
1 \\
1\end{array}$ & $\begin{array}{l}5 / 11 \\
2 / 4 \\
2 / 4 \\
0 / 1 \\
0 / 1 \\
1 / 1\end{array}$ & & \\
\hline $\begin{array}{l}\text { Fusobacterium species } \\
F . \text { nucleatum } \\
\text { F. necrophorum } \\
\text { F. mortiferum }\end{array}$ & $\begin{array}{l}3 \\
1 \\
1 \\
1\end{array}$ & $\begin{array}{l}0 / 3^{\dagger} \\
0 / 1 \\
0 / 1 \\
0 / 1\end{array}$ & & \\
\hline Veillonella species & 1 & $0 / 1$ & & \\
\hline Peptostreptococcus asaccharolyticus & 1 & $0 / 1$ & & \\
\hline Untypable & 1 & $0 / 1$ & & \\
\hline Total & 51 & $12 / 33$ & 12 & $3 / 7$ \\
\hline
\end{tabular}

*Number of deaths/total number of patients with the isolate

tone patient had polymicrobial anaerobic sepsis with $B$. fragilis and $F$. mortiferum.

AAVAMC patients were men. Data summarizing the clinical presentation, selected laboratory findings, and clinical complications in cases of anaerobic bacteremia are summarized in Table II. Although a majority of bacteremic patients were febrile, only one fifth presented with septic shock. A surgical or invasive diagnostic procedure preceded the development of bacteremia in $35 \%$ of the patients. Moreover, since many of the patients had undergone recent contaminated or clean-contaminated surgery, cultures from other sites, such as abscesses or wounds, were frequently positive for anaerobic organisms. Direct complications of anaerobic bacteremia were uncommon. No metastatic abscesses were found, and only two cases of nonseptic thrombophlebitis were diagnosed.

For nearly all of the patients, there was an obvious source for the anaerobic bacteremia (Table III). The putative portals of entry correlated well with those body surfaces on which anaerobes make up the predominant flora, i.e., the gastrointestinal tract, the female genitourinary tract, skin, and oral mucosa. Two thirds of bacteremic patients had obvious intra-abdominal processes that compromised the intestinal mucosal barrier. In a majority of these cases, the structural integrity of the abdominal viscera was compromised, either requiring surgical repair or as a result of a surgical procedure. In no case was the abdominal source occult. Among six patients with skin as the presumed portal of entry, there were three cases of spontaneous clostridial myonecrosis and two cases of a forearm abscess at an injection site in an intravenous drug abuser. The bacteremic source in these cases was also immediately apparent. Other sources for anaerobic bacteremia (e.g., genitourinary, oral) were decidedly rare.

\begin{tabular}{|c|c|c|}
\hline TABLE II & & \\
\hline $\begin{array}{l}\text { Clinical Characteristics of Anaerobic Bact } \\
\text { July } 1987 \text { to December } 1988\end{array}$ & 'emia, UMH and $A$ & AMC, \\
\hline & $\begin{array}{l}\text { No. of Patients/ } \\
\text { Total* }\end{array}$ & $\%$ \\
\hline $\begin{array}{l}\text { Clinical presentation } \\
\text { Fever ( } 38.1^{\circ} \mathrm{C}\left[>100.5^{\circ} \mathrm{F}\right] \text { ) } \\
\text { Localized anaerobic infection } \\
\text { Postoperative fever } \\
\text { Septic shock (SBP }<80 \mathrm{~mm} \mathrm{Hg} \text { ) }\end{array}$ & $\begin{array}{r}22 / 34 \\
16 / 40 \\
13 / 37 \\
8 / 40\end{array}$ & $\begin{array}{l}65 \\
40 \\
35 \\
20\end{array}$ \\
\hline $\begin{array}{l}\text { Laboratory findings } \\
\left.\text { Leukocytosis (WBC }>10,000 / \mathrm{mm}^{3}\right) \\
\text { Hyperbilirubinemia }(>3.0 \mathrm{mg} / \mathrm{dL})\end{array}$ & $\begin{array}{r}26 / 35 \\
7 / 20\end{array}$ & $\begin{array}{l}74 \\
35\end{array}$ \\
\hline $\begin{array}{l}\text { Complications } \\
\text { Thrombophlebitis } \\
\text { Pericarditis } \\
\text { Metastatic abscess }\end{array}$ & $\begin{array}{l}2 / 40 \\
1 / 40 \\
0 / 40\end{array}$ & $\begin{array}{l}5.0 \\
2.5 \\
\end{array}$ \\
\hline
\end{tabular}

$\mathrm{SBP}=$ systolic blood pressure; $\mathrm{WBC}=$ white blood cell count.

* Number of patients with the indicated clinical feature/total number of patients from whose chart evaluable information coutld be ascertained concerning the indicated feature. 


\begin{tabular}{ll}
\hline TABLE III & \\
Source of Anaerobic Bacteremia, by Portal of Entry & \\
\hline & No. \\
\hline Gastrointestinal tract & 25 \\
Perforated bowel & 7 \\
Postoperative abscess or wound infection* & 6 \\
Gl neoplasms & 3 \\
Hepatic cirrhosis & 2 \\
Biliary tract infections & 2 \\
Pyogenic hepatic abscess & 1 \\
Crohn's disease exacerbation & 1 \\
Ischemic bowel & 1 \\
Diverticulitis (seeding pericardium) & 1 \\
Severe mucositis after Ara-C & 1 \\
\hline Skin & 6 \\
Clostridial myonecrosis & 3 \\
Forearm abscess (IV drug user) & 2 \\
Diabetic with dry gangrene & 1 \\
\hline Genitourinary tract & 3 \\
Prostatic abscess with penile gangrene & 1 \\
Endometritis & 1 \\
Infected cervical cancer & 1 \\
\hline Oral & 1 \\
Periapical abscess & 1 \\
\hline Uncertain & 5 \\
Ovarian cancer with fever and neutropenia & 1 \\
Acute lymphocytic leukemia with fever and neutropenia & 1 \\
CVA/aspiration pneumonia & 1 \\
Lymphoma ${ }^{\dagger}$ & 1 \\
Hemodialysis patient ${ }^{\dagger}$ & 1 \\
\hline
\end{tabular}

$\mathrm{Gl}=$ gastrointestinal; $\mathrm{Ara}-\mathrm{C}=$ cytosine arabinoside; $\mathrm{IV}=$ intravenous; $\mathrm{CVA}=$ cerebrovascular accident.

*Includes one patient with a postoperative anastomotic leak and one patient with an inadvertent intraoperative enterotomy.

† Medical record not available for review.

In $35(88 \%)$ of the $40 \mathrm{UMH}$ cases, a source for the anaerobic blood isolate was easily discernable. Among the patients with uncertain sources for bacteremia, two developed anaerobic bacteremia in association with fever and neutropenia during cancer chemotherapy, but neither had a clinically apparent focus of anaerobic infection. One of these two had ovarian carcinoma, and blood cultures obtained during a febrile, neutropenic episode grew an untypable gram-negative, anaerobic bacillus. A third patient was known to have lymphoma, but the medical records pertinent to the episode of anaerobic bacteremia were not available. One patient had a cerebrovascular accident complicated by multiple episodes of aspiration pneumonia; the patient sustained a fall at a chronic care facility and subsequently developed respiratory distress and Bacteroides uniformis bacteremia (a possible oral source). The remaining patient was undergoing hemodialysis and developed $B$. fragilis bacteremia without a clear portal of entry.

\section{Outcome of Anaerobic Bacteremia}

Fifteen patients $(38 \%)$ with anaerobic bacteremia died. All 15 patients who died had either Bacte- roides or Clostridium bacteremia. Ten patients had Bacteroides bacteremia ( $42 \%$ mortality); five patients had Clostridium bacteremia ( $45 \%$ mortality). Although anaerobic bacteremia is associated with a high fatality rate, anaerobic sepsis was not the immediate cause of death in most patients. Septic shock occurred in eight patients (20\%); however, five of these patients had a fatal outcome. In only one patient was an anaerobe cultured from the blood within hours of death. This patient had acute lymphoblastic leukemia and presented to the UMH Emergency Room with signs of acute abdomen and septic shock and was found to have had a bowel perforation and C. septicum bacteremia. He had a cardiac arrest as antibiotics and vasopressors were being administered. Among the four other patients who died with septic shock, none had anaerobes isolated from their blood cultures within 24 hours of death.

Apart from the septic shock, the only other possible predictor of mortality in this study was the source of bacteremia. Among the 25 patients with a gastrointestinal source of bacteremia, $12(48 \%)$ died, whereas only three $(20 \%)$ of the 15 patients with other (or unknown) sources died; however, this difference was not statistically significant. Approximately half ( 19 of 40 ) of the patients had some form of cancer at the time of their bacteremia, but this diagnosis did not confer a significantly worse prognosis; mortality was $42 \%$ in cancer patients and $33 \%$ in noncancer patients.

All 15 fatal cases were treated with antimicrobial agents that have broad activity against anaerobes isolated at our hospitals (i.e., metronidazole, clindamycin, imipenem, or ticarcillin-clavulanate). Antibiotic susceptibility data were available for six of the 15 fatal cases, and, in all six cases, the isolate was sensitive to the antibiotic used. We conclude, therefore, that the choice of inappropriate or ineffective antibiotics was not a factor that contributed to mortality in this series of patients.

\section{Impact of Anaerobic Bacteremia on Patient Management}

We were interested to know what effect the diagnosis of anaerobic bacteremia had on the care of these patients in order to assess the practical utility of routine anaerobic blood cultures. We, therefore, reviewed the records to determine whether the positive culture report resulted in a change in the management of the patient, and if so, whether the change significantly altered the clinical course and outcome.

By retrospective chart review, we could establish with certainty in 29 of the 33 UMH cases and all seven of the AAVAMC cases whether or not antibi- 
otics with broad activity against anaerobes were administered empirically (i.e., clindamycin, metronidazole, $\beta$-lactams with extensive anti-anaerobic activity). In 30 of these 36 evaluable cases, empiric antibiotic therapy directed against anaerobes was used. Albeit in retrospect, we judged that 28 of the 30 patients who received this therapy, and five of the six patients who did not receive empiric coverage had initial clinical presentations that typically evoke suspicion of anaerobic infection.

In only three cases did the culture result in a change in therapy that affected patient management. In the first case, a patient with hepatic cirrhosis developed right upper quadrant abdominal pain, fever, and increased abdominal girth; he was treated empirically with ceftriaxone alone. When his blood cultures became positive for $B$. thetaiotaomicron/ovatus, metronidazole was added to his treatment regimen. In the second case, the patient sustained an inadvertent enterotomy during a nephrectomy for renal cell carcinoma. The patient was treated intraoperatively and postoperatively with cephalothin and gentamicin, but after the blood cultures became positive for $B$. thetaiotaomicron/ovatus, therapy was changed to include metronidazole. The third patient was a febrile, neutropenic patient with acute lymphoblastic leukemia who had metronidazole added to empiric therapy with ceftazidime and tobramycin when the blood culture grew Clostridium ramnosum. The patient tolerated the anaerobic bacteremia well, as she was clinically improving without definitive anaerobic coverage for the 5 days required to grow the isolate from culture. Indeed, the anaerobic isolate may have been a contaminant in this case; however, since the culture result prompted therapeutic action on the part of the physician in charge, the isolate was classified as significant by our predetermined criteria.

In summary, blood culture results rarely led to the initiation of specific antibiotic therapy for anaerobes, since most patients had an obvious source for their infection and were treated with anaerobic coverage empirically. In two of the cases in which a significant therapeutic change was made (the first two cases summarized above), the clinical settings were quite compatible with the eventual isolation of an anaerobic bacterium from blood. It is not possible, in a retrospective analysis, to determine whether the blood culture report influenced the choice or duration of antibiotic therapy that had been begun empirically.

\section{COMMENTS}

Since cultivation of blood for obligate anaerobes was first evaluated in 1938 [11], the frequency and incidence of bacteremia have been difficult to establish. Although a series of frequently cited studies from the Mayo Clinic in the early 1970s reported the frequency of obligate anaerobes in positive blood cultures to be $11.2 \%$ to $27 \%[3,5,12,13]$, other contemporary studies reported much lower isolation rates [14-16]. Finegold [17] reviewed the literature in 1975 and suggested that a generalized frequency might be $5 \%$ to $15 \%$ of all bacteremias. Since 1975 , several more research articles on the epidemiology of bacteremia in general hospital populations have been published in which the frequency or incidence of anaerobic organisms was measured or can be ascertained. In these more recent experiences, anaerobes still represent $1.8 \%$ to $17.3 \%$ of all positive cultures (Table IV). However, the Mayo Clinic reported a decrease in the frequency of anaerobic bacteremia in recent years. The frequency shown in Table IV represents the cumulative experience over 10 years; however, in data summaries from individual years, anaerobes accounted for $17.5 \%$ of microorganisms recovered from blood cultures in 1974, but only $7.4 \%$ of the positive cultures in 1984 [26]. Most of the decline was due to a decrease in the isolation of Bacteroides sp. Comparable observations were made in two more recent studies. A hospital-based study from New York City showed a decline in the proportion of blood cultures positive for Bacteroides sp. from $5.5 \%$ in 1981 to $1.7 \%$ in 1988 [29]. Similarly, a nationwide, voluntary blood culture surveillance system in the United Kingdom reported a decline in the frequency of Bacteroides isolates from $5.8 \%$ in 1975 to $3.5 \%$ in 1980 [37].

Although it is possible that the infrequent isolation of anaerobes reported in this study reflects relatively insensitive culture methodology, we consider this explanation unlikely, since comparable data were obtained from two independent hospital laboratories that use different blood culture systems. It is also possible that the lower frequency is artifactual, resulting from lower rates of anaerobic contaminants or higher rates of either significant or contaminant isolates of aerobic or facultative bacteria in our hospitals. However, a comparison of the incidence rates of clinically significant anaerobic bacteremia belies this notion, since these rates are unaffected by other differences in blood culture isolations. Using a much more stringent definition of "clinical significance" than ours, Wilson and coworkers [12] found an incidence of 1.22 cases of significant anaerobic bacteremia per 1,000 admissions to the Mayo Clinic in the 1970 s, twice the rates in our study.

On these grounds, we believe that the relatively low frequency and incidence of anaerobic bacteremia in our hospitals represent real, and not artifac- 


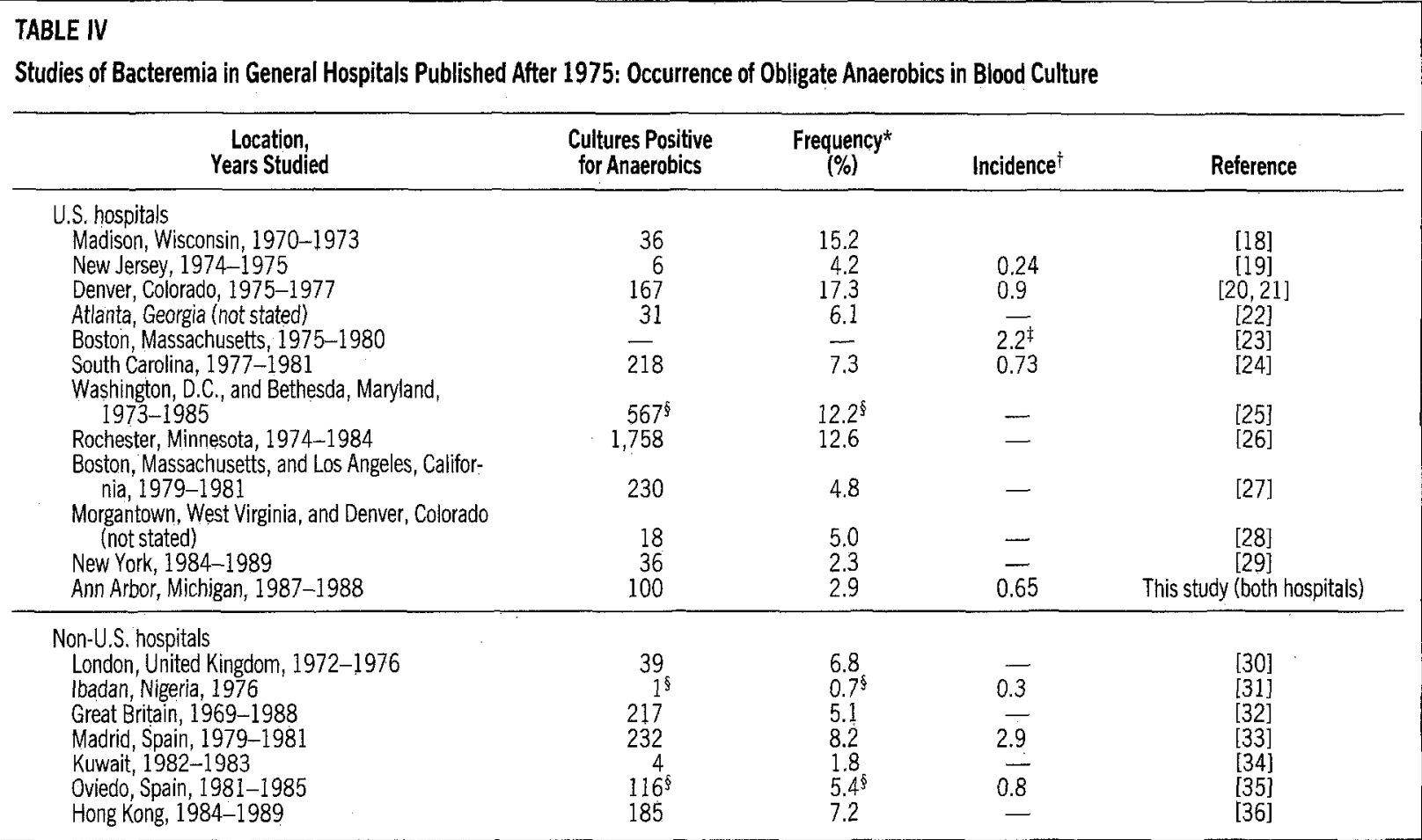

* Percent of all positive blood cultures with obligate anaerobic isolates.

tNumber of episodes of significant anaerobic septicemia per 1,000 hospital admissions.

† only Bacteroides species, Fusobacterium species, and ariaerobic cocci included in data.

§These studies reported the number of patients with anaerobic bacteremia and the proportion of bacteremic patients with anaerobes instead of culture data.

tual, differences with past reports from other hospitals. We cannot compare our current results with those obtained in earlier years, since the culture techniques used prior to the 1980 s were significantly different at both hospitals. However, our data, taken in context with those from the Mayo Clinic and from New York, provide strong grounds for speculation that the incidence of anaerobic bacteremia has actually decreased since the 1970s. One may ask whether our patient population is intrinsically at less risk of anaerobic bacteremia, or whether some aspects of local medical practice might have influenced the occurrence or ascertainment of these cases. We favor the latter notion, since there is no epidemiologic or geographic reason to accept the former. Indeed, the use of antimicrobial agents may well explain the differences. Agents used primarily for their activity against anaerobes, such as clindamycin and metronidazole, are among the most frequently prescribed antibiotics at our institutions, and many of the new broad-spectrum antibiotics that are used empirically in the treatment of nosocomial infections have excellent activity against $\beta$ lactamase-producing Bacteroides species. Since these antibiotics are generally viewed as safe and effective, their use for early or empiric therapy may prevent some bacteremias or make physicians less prone to draw cultures when a focal source of infection is known and has been cultured. Also, the adop- tion of new antibiotics for surgical prophylaxis may have affected the incidence of postoperative anaerobic bacteremia. Unpublished data from the UMH indicate a decline in postoperative infection following colectomy during the late 1970 s coincident with the adoption of cefoxitin in place of cefazolin for surgical prophylaxis (R. Fekety, personal communication).

Although the incidence of anaerobic bacteremia may have declined in recent years, the clinical features of bacteremic patients are the same. As Gorbach and Bartlett [38] pointed out, "A basic fact of our tenuous coexistence with our environment is that 'life on man' is predominantly anaerobic." It is, therefore, not surprising that the clinical manifestations of anaerobic bacteremia are predictable given the known niches of these organisms "on man" and that the vast majority of cases are associated with gastrointestinal pathology. About two thirds of the cases had a gastrointestinal focus as the portal of bacterial entry. Moreover, we found that in $92 \%$ of the retrospectively evaluable cases, the clinical presentation or the nature of the underlying illness suggested possible involvement by anaerobic pathogens. In fact, when empiric antimicrobial therapy was used, broad activity against anaerobes was included in all but two instances.

Despite advances in antimicrobial therapy, the mortality associated with anaerobic bacteremia was 
high in our study. Earlier studies typically reported lower case-fatality rates ( $25 \%$ to $35 \%$ ) for anaerobic bacteremia $[12,21,38,39]$. It has been suggested that the relatively low mortality may be attributable to the significant proportion of obstetric and gynecologic patients in these patient groups [21]. Chow and Guze [39] reviewed reports of Bacteroides bacteremia in 1974 and found that obstetrical patients accounted for $20 \%$ to $30 \%$ of the cases but less than $1 \%$ of the mortality. In our series, only 1 of 40 patients was an obstetric patient, perhaps reflecting the recognition and early treatment of anaerobic infections in these patients. In contrast to earlier studies, nearly half of our patients had some form of malignancy. Patients with malignancies are at greater risk of mortality from bacteremia in general [21] and with Bacteroides in particular $[39,40]$. Given the population differences, it is likely that the relatively high case-fatality rate in our study reflects the severity of the precipitating pathologic processes. In our hospitals, anaerobic bacteremia can be viewed as a potential indicator of a severe underlying disorder, rather than as a primary pathologic process.

Mortality did not appear to be due to a failure of anti-anaerobic antibiotic therapy per se. Our review showed that those patients who died had all received adequate antimicrobial therapy before death. Given the importance of surgical intervention in many anaerobic infections, the feasibility and timing of surgery might have been more relevant predictors of outcome; however, these aspects of care could not be evaluated accurately in our retrospective study.

In considering the overall impact of the diagnosis of anaerobic bacteremia at our institutions, we conclude that the isolation of obligate anaerobes from the blood may signal the presence of a serious underlying condition, usually in the gastrointestinal tract. However, cultivation of clinically significant anaerobes from the blood is an infrequent event in our hospitals. Moreover, anaerobic bacteremias typically occur in association with recognized, predisposing clinical conditions, and, consequently, their detection rarely results in a change of patient management or clinical outcome.

\section{ADDENDUM}

As this article went to press, data showing declining rates of anaerobic bacteremia from two other sources appeared in the published literature. The proportion of bacteremias caused by anaerobes at the Mayo Clinic (Rochester, Minnesota) reportedly declined from $20.7 \%$ in 1974 to $10.5 \%$ in 1988 [41]. Similarly, the proportion of nosocomial bacteremias caused by anaerobes declined from $7.2 \%$ during the years 1969 to 1979 to $4.5 \%$ during the years 1980 to 1990 at Guy's and St. Thomas' Hospitals, London, United Kingdom [42].

\section{ACKNOWLEDGMENT}

We thank F. Robert Fekety, M.D., J.R.C. Crump, and B.I. Eisenstein for helpful suggestions concerning the manuscript. We also thank members of the microbiology laboratories at the UMH and AAVAMC for their cooperation in providing the blood culture data.

\section{REFERENCES}

1. Attebery HR, Finegold SM. Combined screw-cap and rubber-stopper closure for Hungate tubes (pre-reduced, anaerobically sterilized roll tubes and liquid media). Appl Microbiol 1969; 18: 558-61.

2. Zabransky RJ. Isolation of anaerobic bacteria from clinical specimens. Mayo Clin Proc 1970; 45: 256-64.

3. Washington JA II. Comparison of two commercially available media for detection of bactercmia. Appl Microbiol 1971; 22: 604-7.

4. Martin WJ. Practical method for isolation of anaerobic bacteria in the clinical laboratory. Appl Microbiol 1971; 22: 1168-71.

5. Washington JA II. Evaluation of two commercially available media for detection of bacteremia. Appl Microbiol 1972; 23: 956-9.

6. Martin WJ. Isolation and identification of anaerobic bacteria in the clinical laboratory. Mayo Clin Proc 1974; 49: 300-8.

7. Kasper DL. Infections due to mixed anaerobic organisms. In: Wilson JD, Rraunwald $F$, Isselbacher $\mathrm{KJ}$, edifors. Harrison's principles of internal medicine. 12th ed. New York: McGraw-Hill, 1991: 584-90.

8. Simon HB, Swartz MN. Anaerobic infections. In: Rubenstein E, Federman DD, editors. Medicine. New York: Scientific American, 1989; 7: $\mathrm{V}: 1-17$.

9. Eykyn SJ, Phillips I. Anaerobic bacteremia. In: Weatherall DJ, Ledingham JGG, Warrell DA, editors. Oxford textbook of medicine, vol 1. 2nd ed. Oxford: Oxford University Press, 1987: 224-321.

10. Finegold SM, George WL. Infections caused by nonclostridial anaerobes. In: Kelley WN, editor. Textbook of internal medicine. Philadelphia: JB Lippincott, 1989: 1562-6.

11. Rosenow EC II, Brown AE. Septicemia: a review of cases, 1934-1936, inclusive. Proc Staff Mcet Mayo Clin 1938; 13: 89--93.

12. Wilson WR, Martin WJ, Wilkowske CJ, Washington JA II. Anaerobic bacter emia. Mayo Clin Proc 1972; 47: 639-46.

13. Washington JA H. Martin W.J. Comparison of three blood culture media for recovery of anaerobic bacteria. Appl Microbiol 1973; $25: 70-1$.

14. DuPont HL, Spink WW. Infections due to gram-negative organisms: an analysis of 860 patients with bacteremia at the University of Minnesota Medical Center, 1958-1966. Medicine (Baltimore) 1969; 48: 307-32.

15. Rosner R. A quantitative evaluation of three blood culture systems. Am .I Clin Pathol 1972; 57: 220-7.

16. Shanson DC. Bacteroides in the blood. Lancet 1973; 1: 147-8.

17. Finegold SM. Anaerobic bacteria in human disease. New York: Academic Press, 1977: 182-201.

18. Scheckler WE. Septicemia in a community hospital, 1970 through 1973. JAMA 1977; 237: 1938-41.

19. Setia U, Gross PA. Bacteremia in a community hospital: spectrum and mortality. Arch Intern Med 1977; 137: 1698-1701.

20. Weinstein MP, Reller LB, Murphy JR, Lichtenstein KA. The clinical significance of positive blood cultures: a comprehensive analysis of 500 episodes of bacteremia and fungemia in adults. 1. Laboratory and epidemiologic observations. Rev Infect Dis 1983; 5: 35-53.

21. Weinstein MP, Murphy JR, Reller LB, Lichtenstein KA. The clinical significance of positive blood cultures: a comprehensive analysis of 500 episodes of bacteremia and fungemia in adults. II. Clinical observation, with special reference to factors influencing prognosis. Rev Infect Dis 1983; 5: 54-70.

22. Coleman RM, Laslie WW, Lambe DW Jr. Clinical comparison of aerobic, hypertonic, and anaerobic culture media for the radiometric detection of bacteremia. J Clin Microbiol 1976; 3: 281-6.

23. Henry S, DeMaria A II, McCabe WR. Bacteremia due to Fusobacterium species. Am J Med 1983; 75: 225-31.

24. Bryan CS, Reynolds KL, Metzger WT. Bacteremia in diabetic patients: comparison of incidence and mortality with nondiabetic patients. Diabetes Care 
1985; 8: 244-9.

25. Brook I. Anaerobic bacterial bacteremia: 12-year experience in two military hospitals. J infect Dis 1989; 160: 1071-5.

26. Dorsher CW, Wilson WR, Rosenblatt JE. Anaerobic bacteremia and cardiovascular infections. In: Finegold SM, George WL, editors. Anaerobic infections in humans. San Diego: Academic Press, 1989: 289-310.

27. Martin WJ, Wilhelm PA, Bruckner D. Recovery of anaerobic bacteria from vented blood-culture bottles. Rev Infect Dis 1984; 6 Suppl 1: S59-61.

28. Reimer LG, McDaniel JD, Mirrett S, Reller LB, Wang WL. Controlled evaluation of supplemented peptone and Bactec blood culture broths for the detection of bacteremia and fungemia. J Clin Microbiol 1985; 21: 531-4.

29. Neu HC, Fracaro M, Bopp H, O'Keefe M, O'Connor J. Bacteraemia-a New York perception. J Antimicrob Chemother 1990; 25 Suppl C: 107-113.

30. Abeysundere RL, Bradley JM, Chipping P, Rogers BT, Noone P. Bacteremia in the Royal Free Hospital, 1972-1976. J Infect 1979; 1: 127-38.

31. Alausa KO, Montefiore D, Sogbetun AO, Ashiru JO, Onile BA, Sobayo E. Septicaemia in the tropics; a prospective epidemiological study of 146 patients with a high case fatality rate. Scand J Infect Dis $1977 ; 9$ : 181-5.

32. Eykyn S.J, Gransden WR, Phillips I. The causative organisms of septicaemia and their epidemiology. J Antimicrob Chemother 1990; 25 Suppl C: 41-58.

33. Bouza E, Reig M, Garcia de la Torre M, et al. Retrospective analysis of two hundred and twelve cases of bacteremia due to anaerobic microorganisms. Eur $\mathrm{J}$ Clin Microbiol 1985; 4: 262-7.

34. Elhag KM, Mustafa AK, Sethi SK. Septicaemia in a teaching hospital in Kuwait-I. Incidence and aetiology. J Infect 1985; 10: 17-24.

35. Vazquez F, Mendez FJ, Perez F, Mendoza MC. Anaerobic bacteremia in a general hospital; retrospective five-year analysis. Rev Infect Dis 1987; 9: $1038-43$.

36. French GL, Cheng AF, Duthie R, Cockram CS. Septicaemia in Hong Kong. J Antimicrob Chemother 1990; 25 Suppl C: 115-25.

37. Young SEJ. Bacteremla 1975-1980: a survey of cases reported to the PHLS Communicable Disease Surveillance Centre. J Infection 1982; 5: 19-26.

38. Gorbach SL, Bartlett JG. Anaerobic infections. N Engl J Med 1974; 290 : 1177-84, 1237-45, 1289-94.

39. Chow AW, Guze LB. Bacteroidaceae bacteremia: clinical experience with 112 patients. Medicine (Baltimore) 1974; 53: 93-126.

40. Felner JM, Dowell VR. "Bacteroides" bacteremia. Am J Med 1971; 50 : 787-96.

41. Dorsher CW, Rosenblatt JE, Wilson WR, Ilstrup DM. Anaerobic bacteremia: decreasing rate over a 15 year period. Rev Infect Dis 1991; 13: 633-6.

42. Gransden WR, Eykyn SJ, Phillips I. Anaerobic bacteremia: declining rate over a 15-year period. Rev Infect Dis 1991; 13: 1255-6. 